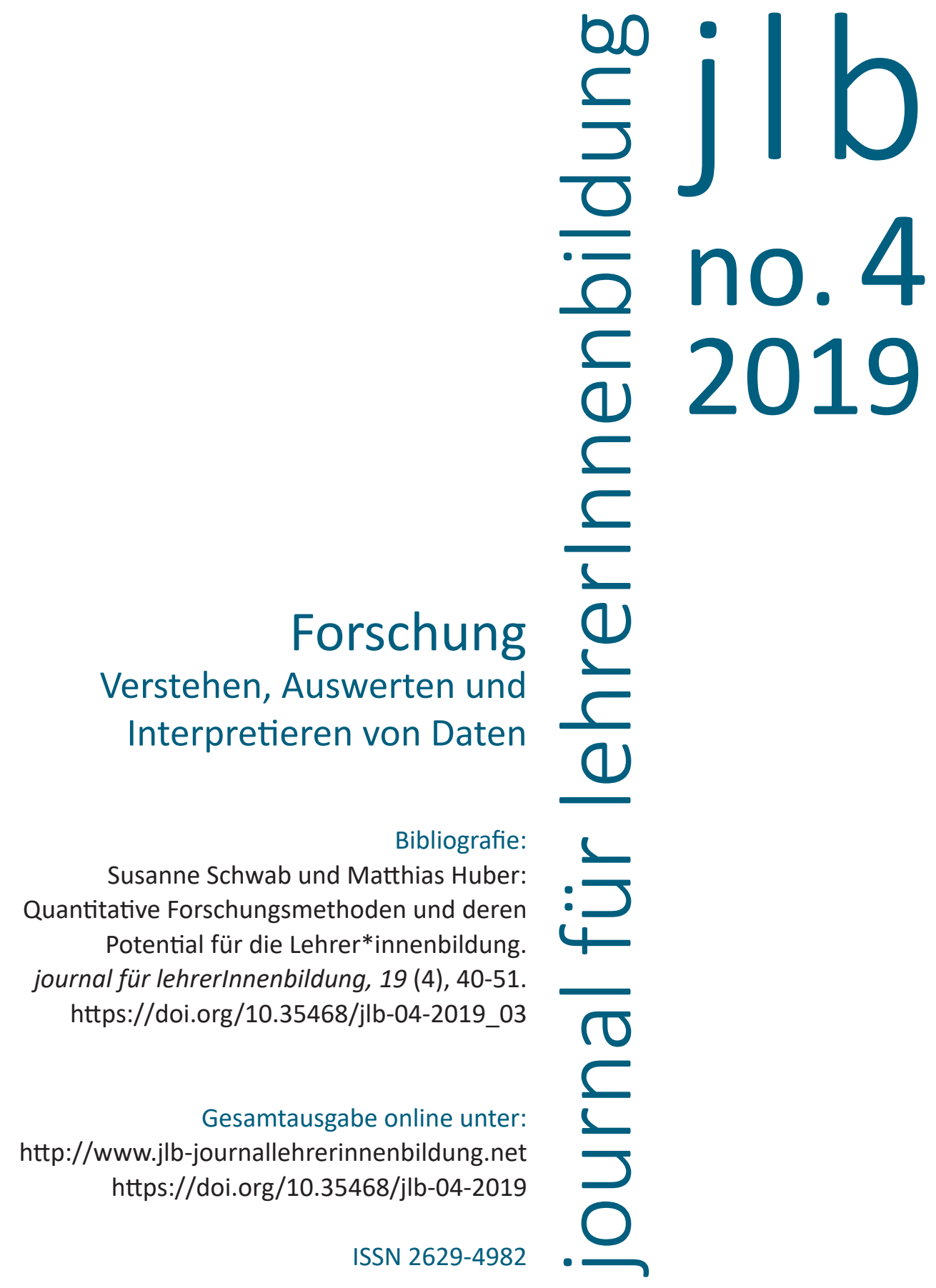


03

Susanne Schwab und Matthias Huber

\section{Quantitative Forschungs- methoden und deren Potenzial für die Lehrer*innenbildung}




\section{Einleitung}

„Dem Pädagogik-Paket fehlt es an wissenschaftlicher Evidenz" heißt es seitens der Österreichischen Gesellschaft für Forschung und Entwicklung im Bildungswesen (ÖFEB, 2018) in einer Aussendung zum geplanten Pädagogik-Paket der Bundesregierung Österreichs. Diese Aussage ist nicht weiter verwunderlich, sagte doch Österreichs ehemaliger Bildungsminister Faßmann, dass nicht alle politischen Entscheidungen wissenschaftlich fundiert sind. Wenngleich man nun von angehenden Lehrkräften am Ende der Ausbildung nicht erwarten wird, dass diese selbst Politiker*innen werden, so erscheint es dennoch als essentiell, dass Lehrkräfte, welche letztendlich oftmals die ausführenden Kräfte bildungspolitischer Entscheidungen sind, verstehen, welche Bedeutung wissenschaftliche Evidenz für ihre Tätigkeit hat. Hierbei ist nicht nur das Verstehen, wie (quantitative) Bildungsforschung missbraucht wird bzw. werden kann, sondern auch das Wissen über die Konsequenzen des Ignorierens von wissenschaftlichen Ergebnissen essentiell. Empirische Forschung schafft für Lehrkräfte Sicherheit (Hellmich \& Löper, im Druck), wenn es beispielsweise um die konkrete Unterrichtsgestaltung, die Entwicklung von Förderkonzepten, die eigene Schulund Unterrichtsentwicklung (Wiesner, Schreiner, Breit \& George, 2018) oder aber um die Persönlichkeitsentwicklung der Schüler*innen (Huber, 2019) geht.

\section{Potenzial quantitativer Forschungsmethoden im schulischen Kontext}

Schulsysteme unterliegen immer nationalen und internationalen Veränderungen unterschiedlichen Ausmaßes. Beispielsweise kann schulische Inklusion als ein europaweites Bildungsziel betrachtet werden (Schwab, 2019). Diese Entwicklungen sollten bestenfalls auf wissenschaftlicher Fundierung basieren, um tatsächlich zu wissen, dass der gemeinsame Unterricht auch die bestmögliche Förderung aller Schüler*innen bewirkt. Insofern geht es darum, zu wissen was wirkt. Insbesondere quantitative Studien können hierbei unterstützen, dass Wissen Praxis schafft.

In Österreich lässt sich zudem in den letzten Jahren verstärkt ein Trend zur "Kompetenzkultur" beobachten. Dies ist an der Teilnehme 
an internationalen Vergleichsstudien (PISA, PIRLS, TIMSS), den nationalen Bildungsstandardüberprüfungen wie auch an der Einführung von nationalen Bildungsstandards (z. B. Zentralmatura) ersichtlich. Im Zuge der internationalen Schulleistungsstudien wurde es möglich zu wissen, wo die eigenen Schüler*innen im Vergleich zu jenen anderer Länder mit Blick auf spezifische Schulfähigkeiten stehen. Neben dem absoluten Vergleich von Leistungen können diese Studien jedoch auch Rückschlüsse auf Wirkungsmechanismen zulassen. Beispielsweise ist dem aktuellen Bericht der OECD (2018) zu entnehmen, welche Länder im Zuge der PISA-Erhebungszyklen Equity-Verbesserungen erzielten. Durch diesen evidenzbasierten und output-orientierten Ansatz einer Qualitätssicherung (Altrichter \& Maag Merki, 2016) gewinnt die Gestaltung erfolgreicher Bildungsprozesse neue Formen. Das Potenzial dieser Veränderungen ist jedoch nur gewährleistet, wenn auch der Transfer in die Praxis gegeben ist und hierzu bedarf es einer systematischen und praxisorientierten Vermittlung von Methodenkompetenz während des gesamten Studiums.

\section{Aspekte bei der Vermittlung quantitativer Methoden}

Stelter und Miethe (2019) stellen in ihrer aktuellen Studie fest, dass in Bezug auf die forschungsmethodische Ausbildung bei Lehrkräften im Vergleich zu jenen der Erziehungs- und Bildungswissenschaft ein Weiterbildungsbedarf besteht. Um das Potenzial (quantitativer) Methoden für die Lehrer*innenbildung maximal ausschöpfen zu können, stellt sich allerdings die Frage, was nun zukünftigen Lehrkräften tatsächlich vermittelt werden soll. Grundsätzlich bedarf es eines generellen Wissens um die vorhandene Methodenvielfalt sowie um die Passung von Fragestellung und Methode. Hierbei steht u. a. im Fokus, dass Studierende (sowie Lehrkräfte) die Logik quantitativer Forschung erschließen müssen, um deren Bedeutung und Anwendung kritisch einschätzen zu können. Dazu ist zwar die Vermittlung von ausgewählten Grundlagen der quantitativen Forschung (z. B. Testtheorie; Moosbrugger \& Kelava, 2012) unumgänglich, allerdings ist dabei vielmehr der Kontext eines kritisch reflexiven Wissens entscheidend. So ist die Kenntnis, dass die Reliabilität nach Cronbach's Alpha .8 betragen sollte nicht ausreichend, wenn die Bedeutung schlechter Gütekriterien 
für die Interpretation der Daten unbekannt ist. Insofern scheint in der Lehrer*innenbildung weniger das "WAS" für die Vermittlung bedeutend, sondern vielmehr das "WIE“: „Wie kann eine Lehrkraft verstehen, welche Bedeutung eine Studie für die eigenen Entscheidungen in der Praxis hat?" "Warum ist es wichtig, dass eine Studie ,sauber" durchgeführt wird?" "Wie und warum nütze ich das gewonnene Wissen?" Wenn eine Lehrkraft beispielsweise Leistungstestergebnisse ihrer Schüler*innen vergleicht, so sollten Fragen nach der konkreten Förderung im Vordergrund stehen, $d$. $h$. es geht um Leistungspotenziale und ihre prospektive Umsetzung und nicht um Fragen nach Statusdiagnostik und entsprechenden Kategorisierungen.

\section{Chancen quantitativer Forschungsmethoden in der Schulforschung und Lehrer*innenbildung}

Die Analyse der Komplexität pädagogischer Interaktionen geht mit einer Vielzahl an methodologischen Schwierigkeiten und forschungsmethodischen Unsicherheiten einher. Nicht nur die Passung von Methode und Gegenstand, sondern im Besonderen die Berücksichtigung forschungsethischer Gesichtspunkte, (wie bspw. Anonymität, Freiwilligkeit, Transparenz oder Vertraulichkeit etc.) bei gleichzeitiger Wahrung der pädagogischen Aufgabe der Vermittlung macht den Topos Schule, unabhängig vom jeweiligen Erkenntnisinteresse, zu einem durchaus problematischen und schwer zu erschließenden Forschungsfeld. Der Einsatz quantitativer Forschungsmethoden ermöglicht dabei durch die Erfassung und Darstellung der Variabilität eines bestimmten Merkmals anhand der definierten Zuordnung von Zahlenwerten ein präzises, objektives und systematisches Vorgehen. Damit ist eine Reihe von Vorzügen sowohl für die Schulforschung $(A)$ als auch für die Lehrer*innenbildung selbst (B) verbunden:

A. Mit Blick auf den schulischen Kontext eröffnet die Fokussierung auf standardisierte Daten die Möglichkeit weitaus größere Schüler*innengruppen zu untersuchen, respektive eine wesentlich größere Stichprobe zu erfassen, und somit ausreichende Häufigkeiten für bestimmte Merkmalkombinationen bereit zu stellen. Dies wiederum erlaubt eine bessere Vergleichbarkeit der Ergebnisse mit anderen Studien sowie die Überprüfung vorab festgelegter 
Hypothesen im Kontext des zu untersuchenden Forschungsgegenstandes, was insbesondere in der empirischen Lehr-Lernforschung eine Notwendigkeit innovativer Theoriebildung darstellt (Jütte, Walber \& Lobe, 2017). Der damit implizierte Anspruch der Quantifizierbarkeit, Messbarkeit und Reproduzierbarkeit statistischer Daten sowie die Reduktion komplexer Fragestellungen und Zusammenhänge auf wenige Aussagen bzw. Zahlenwerte unterstreicht überdies das Potenzial quantitativer Forschungsmethoden für die Schul- und Unterrichtsforschung.

B. Im Kontext der Lehrer*innenbildung sowie hinsichtlich der Vermittlung von Methodenkompetenz im Studium geht der Einsatz quantitativer Methoden zudem mit einer Reihe von Vorzügen einher. Besonders die von der Untersuchungsleitung ausgehenden Einflüsse auf den Forschungsgegenstand sowie die subjektive Perspektive der untersuchenden Person werden weitgehend eliminiert, was den Einsatz und Umgang für Studierende erleichtert (Raithel, 2008). Zudem erhöhen statistische Ergebnisse durch die Reduktion von Information auf das Notwendigste die Klarheit des Denkens und ermöglichen eine bessere Organisation von bestehendem Wissen im Kontext der eigenen Forschungspraxis. Das regelgeleitete und standardisierte Vorgehen quantitativer Analysemethoden erlaubt überdies eine präzise Messung und Auswertung, selbst wenn die Studierenden noch wenig praktische Erfahrung im empirischen Feld sammeln konnten.

\section{Herausforderungen quantitativer Forschungsmethoden in der Schulforschung und Lehrer*innenbildung}

Gleichzeitig muss jedoch darauf hingewiesen werden, dass der Einsatz quantitativer Forschungsmethoden sowohl im Schulkontext (A) als auch in der Ausbildung zukünftiger Lehrkräfte (B) mit einigen Herausforderungen einhergeht:

A. Besonders die zuvor angesprochene Komplexität pädagogischer bzw. sozialer Interaktionen, die sich vorrangig durch die Individualität und Subjektivität der jeweiligen Lehr-Lern-Konstellation und ihrer Protagonist*innen auszeichnet, stellt für viele quantifizieren- 
de Zugänge eine große Schwierigkeit dar (Lamnek, 2010). Denn ein tatsächliches Problem statistischer Daten, wie dies bspw. beim Einsatz von standardisierten Fragebögen immer wieder deutlich wird, ist der damit einhergehende Verlust der subjektiven Sichtweisen der zu untersuchenden Schüler*innen. Damit einher geht der Vorwurf einer fehlenden Praxisnähe statistischer Analysen sowie der Vorwurf, nicht lebensnah zu sein bzw. für die/den konkrete*n Schüler*in mit ihren spezifischen Problemen, Sorgen und Ängsten nur wenig Aussagekraft zu haben. Daran schließt sich eine weitere Einschränkung der rein quantitativen Verfahren an: durch den Anspruch der Standardisierung wird die Eigenart des Forschungsgegenstandes vernachlässigt. Dies wiederum führt zu dem Vorwurf, dass die quantitative Forschung starr und geschlossen agiert und sich nicht weiter um die Bedingungen des Zustandekommens der jeweiligen, zu messenden Merkmale bemüht (Keeves \& Masters, 1999). Die Voraussetzung gleichbleibender Untersuchungsbedingungen und die Kontrolle derselben sowie das Verbot der Modifikation des methodischen Vorgehens während des Forschungsprozesses verstärken diesen Vorwurf, im Besonderen im Kontext von Schule und Unterricht, die sich als Forschungsgegenstände gerade durch ihre Flexibilität und Offenheit charakterisieren.

B. Und auch mit Blick auf die Ausbildung zukünftiger Lehrkräfte finden sich spezifische Problemlagen im Hinblick auf den Einsatz, die Erprobung und Durchführung quantifizierender Verfahren. Besonders auffällig im Kontext universitärer Lehre ist dabei eine, bei den Studierenden früh einsetzende, Form des Messfetischismus. Durch die Überbetonung von Methodologie, Technik, Regelgeleitetheit und Gütekriterien entsteht bei Studierenden die Vorstellung, dass Forschung ein getreues Abbild der Wirklichkeit ermöglicht, sofern Zahlen und Werte als valide, reliabel und objektiv ausgewiesen sind (Leonhart, 2004). Allerdings ist der Glaube an evidenzbasierte Forschung im schulischen Kontext durchaus kritisch zu betrachten, da wissenschaftliche Erkenntnisse oft fälschlicherweise als gesichert und somit als allgemeingültig für alle Schüler*innen, zu jeder Zeit und an jedem Ort verstanden werden (Biesta, 2011). Besonders für die Schulentwicklung und Unterrichtsforschung ist ein solches Wissenschaftsverständnis zukünftiger Lehrpersonen verhängnisvoll. Ein weiteres Problem besteht für Studierende oft darin, durch die vorab konstruierten Hypothesen bereits festzule- 
gen, was in der bevorstehenden Untersuchung relevant ist. Somit wird die eigene Perspektive stark eingeschränkt, was beispielsweise in der thematischen Vorstrukturierung spezifischer Items oder Antwortmöglichkeiten deutlich wird, und es besteht die Gefahr der blinden Reproduktion allgemeiner Annahmen und Thesen.

Abschließend sei noch eine generelle Schwierigkeit deduktiver Erkenntnisgenerierung angesprochen, die selten im Kontext von Schule und Unterricht thematisiert wird, aber ein grundlegendes Problem der quantitativen Forschungslogik verdeutlicht. Denn eine große Gefahr und womöglich die größte Limitation quantitativer Analysen besteht in der vorschnellen Generalisierung von Annahmen. So werden statistische Teilergebnisse, insbesondere in bildungspolitischen Diskursen, gerne dazu verwendet, durch die normative Ausdeutung derselben Meinungsbildung zu betreiben, anstatt spezifische Problemlagen im Bildungsbereich sichtbar zu machen und kritisch zu diskutieren. In den meisten Fällen lassen sich dabei die Normative (wie z. B. Deutschförderklassen verbessern die Integration) mit den zur Meinungsbildung herangezogenen statistischen Ergebnissen (wie z. B. lediglich 32\% der Schüler*innen mit Migrationshintergrund erreichen die Bildungsstandards in der 8. Schulstufe) in keiner Weise zusammenführen (BMBWF, 2019). Hier verlangt es nach einer kritischen, wissenschaftstheoretisch und methodologisch pluralen Perspektive, die als Voraussetzung von Methodenkompetenz im Lehramtsstudium besonderer Aufmerksamkeit bedarf.

\section{Illustration eines Anwendungsbeispiels: Perception of Inclusion Questionnaire}

Ein konkretes Fallbeispiel, welches den Nutzen quantitativer Forschungsmethoden und deren Potenzial für die Lehrer*innenbildung verdeutlicht, ist der Einsatz des Perception of Inclusion Questionnaires (PIQ: www.piqinfo.ch; Venetz, Zurbriggen, Eckhart, Schwab \& Hessels, 2015). Dieser Fragebogen ermöglicht es Lehrkräften, das emotionale Wohlbefinden, das akademische Selbstkonzept und die soziale Inklusion ihrer Schüler*innen (im Alter von 8 bis 16 Jahren) eigenständig zu erfassen. Alle drei Merkmale können damit innerhalb 
von etwa fünf Minuten erfasst werden. Der Fragebogen selbst besteht aus zwölf einfachen Fragen (z. B. Ich gehe gerne in die Schule.), welche entweder vom Schulkind oder vom Jugendlichen selbst, der Lehrkraft oder den Eltern ausgefüllt werden können. Für die Auswertung kann man entweder auf das vorhandene Auswertungstool oder auf die weiteren Auswertungshinweise zurückgreifen. Das Besondere an dem Instrument ist, dass man damit zuverlässig bzw. reliabel (Zurbriggen, Venetz, Schwab \& Hessels, 2019) und zugleich systematisch mehrere Perspektiven erfassen kann.

Methodisch besteht der Vorteil, dass man nicht auf die Vergleichbarkeit mit anderen Stichproben, Mitschüler*innen etc. verzichten muss. Würde man beispielsweise die Frage stellen „Wie geht es dir in der Schule?" so würde dies relativ viel Zeit benötigen, um alle Kinder einer Schulklasse zu befragen. Zudem bestünde die Gefahr, dass jedes Schulkind auf unterschiedliche Facetten des schulischen Wohlbefindens Bezug nimmt.

Als Lehrkraft kann dieses Screeninginstrument also helfen, Schüler*innen mit geringer subjektiv wahrgenommenen emotionalen, akademischen und sozialen Inklusion rasch zu identifizieren und konkret mit einer Förderung bei Zielkindern oder der kompletten Klasse anzusetzen. Dabei gelingt es auf einfache Weise Einblicke in die Schüler*innenperspektive zu erhalten, um spezifische Förderangebote zeitnah bereitzustellen. Venetz, Zurbriggen und Schwab (2019) konnten beispielsweise zeigen, dass die Einschätzung von Lehrkräften und Schüler*innen in Bezug auf diese frei genannten Dimensionen relativ stark voneinander abweicht.

Um das Instrument nun als Lehrkraft im Unterricht einsetzen zu können, braucht es im Zuge der Ausbildung bereits Wissen darüber, wie ein guter Fragebogen konzipiert sein sollte (forschungsmethodisches Wissen über Gütekriterien quantitativer Forschung). Hierfür reicht es nicht aus, aus dem Manual bzw. aus vorliegenden Publikationen Informationen über die Reliabilität und Validität des Instruments entnehmen und einordnen zu können, vielmehr ist ein kritisches Wissen in Bezug auf die Interpretation der gewonnen Daten notwendig. Woher weiß man z. B., ob durch meine Intervention tatsächlich eine Veränderung herbeigeführt wurde? Inwiefern kann man Ergebnisse auf andere Ursachen (z. B. Vorkommnisse in Schule oder Unterricht) zurückführen? Oder: Wozu sind in dem Fragebogen auch negativ formulierte Fragen enthalten? 


\section{Fazit}

Zusammenfassend kann festgehalten werden, dass es in jedem Fall von Vorteil wäre, wenn alle Entscheidungsträger*innen des Bildungssystems und im Besonderen Lehrpersonen eine basale Methodenkompetenz quantitativer Forschungszugänge im Laufe ihrer Ausbildung entwickeln. Hierfür sprechen zumindest die folgenden vier Aspekte:

1. Erstens werden Lehrer*innen dadurch dazu befähigt, Studien der empirischen Bildungsforschung eigenständig aufzuarbeiten und nachzuvollziehen, um einordnen zu können, welche Tragweite bestimmte Ergebnisse (z. B. jene internationaler Vergleichsstudien) im Kontext von Schulentwicklung und Unterrichtsgestaltung auf bildungspolitischer Ebene einnehmen.

2. Zweitens werden Lehrpersonen immer wieder mit Testergebnissen von Schüler*innen konfrontiert (z. B. im Rahmen psychologischer Testverfahren), die einen spezifischen Handlungsbedarf in der individuellen Begleitung und Förderung nahelegen. Besonders hier wäre eine gewisse quantitative Methodenkompetenz von zentraler Bedeutung für das professionelle Selbstverständnis und die Abgrenzung gegenüber anderen Berufsgruppen im Bildungsbereich.

3. Drittens kann es, wie am Beispiel des PIQ verdeutlicht, an manchen Stellen durchaus hilfreich sein, selbstständig quantifizierende Verfahren anzuwenden, um bspw. die eigene Unterrichtsqualität evaluieren oder Kompetenzmessungen eigenständig durchführen zu können, was wiederum die Qualität der eigenen pädagogischen Arbeit verbessert.

4. Und viertens ermöglicht eine im Studium erworbene quantitative Methodenkompetenz Lehrpersonen im Allgemeinen eine schnelle und präzise Untersuchung größerer Stichproben (z. B. mehrerer Klassen), die Prüfung von Hypothesen (z. B. im Kontext der eigenen Lehrevaluation), eine generelle Informationsreduktion (z. B. mit Blick auf spezifische Merkmalausprägungen in einer Klasse), die Kontrolle äußerer Bedingungen (z. B. von Aufmerksamkeit und Konzentration) und den Vergleich mit anderen Studien (z. B. im Rahmen eines aktuellen Schulprogramms oder einer Projektschule). Darüber hinaus schafft die Verwendung von quantitativen Forschungsmethoden eine Distanz zwischen Forscher*innen und Gegenstand. Das hilft die emotionale Involviertheit zu reduzieren. 
Sowohl die eben skizzierten Vorzüge als auch die Gefahren und Herausforderungen quantifizierender Zugänge, wie sie in diesem Beitrag vorgestellt wurden, verdeutlichen, dass es im Kontext methodologischer Überlegungen und im Hinblick auf die Vermittlung von Methodenkompetenz in der Lehrer*innenbildung einer kritischen Perspektive bedarf. Dieser kritische Blick inkludiert eine Sowohl-alsauch-Haltung im Hinblick auf die Frage nach qualitativen und quantitativen Zugängen der Bildungsforschung und versteht Methoden grundsätzlich als Werkzeuge, die je nach Gegenstand und Fragestellung nur dann zum Einsatz kommen, wenn sie tatsächlich und begründet gebraucht werden.

Mit Blick auf aktuelle Entwicklungen in der Schulforschung und Lehrer*innenbildung lässt sich abschließend festhalten, dass innovative Forschung und Lehre sich in erster Linie durch eine plurale und dialektische Grundhaltung auszeichnet und dementsprechend triangulative und multi-methodische Forschungsdesigns die Zukunft der Bildungsforschung darstellen. In diesem Sinne sind quantitative Forschungsmethoden lediglich eine Speiche am Rad jener innovativen Forschungsbemühungen, die das Potenzial haben, Schule und Unterricht sowie die Ausbildung zukünftiger Lehrpersonen nachhaltig zu verbessern.

\section{Literatur}

Altrichter, H. \& Maag Merki, K. (2016). (Hrsg.). Handbuch Neue Steuerung im Schulsystem (2. Aufl.). Wiesbaden: Springer VS.

Biesta, G. (2011). Warum „What works“ nicht funktioniert: Evidenzbasierte pädagogische Praxis und das Demokratiedefizit der Bildungsforschung. In J. Bellmann \& Th. Müller (Hrsg.), Wissen was wirkt. Kritik evidenzbasierter Forschung (S. 95122). Wiesbaden: VS Verlag.

BMBWF (2019). Deutschförderklassen und Deutschförderkurse. Leitfaden für Schulleiterinnen und Schulleiter. Wien: Bundesministerium für Bildung, Wissenschaft und Forschung. Verfügbar unter: https://bildung.bmbwf.gv.at/schulen/unterricht/ba/ deutschfoerderklassen.pdf?6hwy6c [26.08.2019].

Hellmich, F. \& Löper, M. F. (im Druck). Quantitative Zugänge in der Inklusionsforschung. Qualifizierung für Inklusion. Zeitschrift zur Forschung über Aus-, Fort- und Weiterbildung pädagogischer Fachkräfte (Schwerpunktheft „Inklusion: Grundlagen - Theorien - Methodologien").

Huber, M. (2019). Learning Morality? Über die Notwendigkeit (der Vermittlung) von Normen und Werten im Kontext globaler Transformationen. In I. Clems, S. Hornberg \& M. Rieckmann (Hrsg.), Bildung und Erziehung im Kontext globaler Transformationen (S. 193-204). Opladen u. a.: Budrich. 
Jütte, W., Walber, M. \& Lobe, C. (2017). Das Neue in der Hochschullehre. Lehrinnovationen aus der Perspektive der hochschulbezogenen Lehr-Lern-Forschung. Wiesbaden: Springer VS.

Keeves, J. P. \& Masters, G. N. (1999). Issus in Educational Measurement. In J. P. Keeves \& G. N. Masters (Ed.), Advances in Measurement in Educational Research and Assessment (S. 268-282). Oxford: Elsevier Science.

Lamnek, S. (2010). Qualitative Sozialforschung (5. Auf.). Weinheim \& Basel: Beltz.

Leonhart, R. (2004). Lehrbuch Statistik. Einstieg und Vertiefung. Bern: Hans Huber.

Moosbrugger, H. \& Kelava, A. (2012). (Hrsg.). Testtheorie und Fragebogenkonstruktion (2. Aufl.). Heidelberg: Springer.

ÖFEB (2018). Stellungnahme der Österreichischen Gesellschaft für Forschung und Entwicklung im Bildungswesen (ÖFEB) zum geplanten Pädagogik-Paket der Bundesregierung. Verfügbar unter: https://www.oefeb.at/fileadmin/OEFEB_Presse/ OEFEB_Stellungnahme_Paedagogik-Paket_2018.pdf [26.08.2019].

Raithel, J. (2008). Quantitative Forschung. Ein Praxisbuch (2. Auf.). Wiesbaden: Springer VS.

Schwab, S. (2019). Inclusive and Special Education in Europe. Oxford Research Encyclopedia of Education. Retrieved 13 Jun. 2019, from https://oxfordre.com/ education/view/10.1093/acrefore/9780190264093.001.0001/acrefore-9780190 264093-e-1230 [20.10.2019].

Stelter, A. \& Miethe, I. (2019). Forschungsmethoden im Lehramtsstudium - aktueller Stand und Konsequenzen. Erziehungswissenschaft, 58 (30), 25-33.

Venetz, M., Zurbriggen, C., Eckhart, M., Schwab, S. \& Hessels, M. G. P. (2015). The Perceptions of Inclusion Questionnaire (PIQ). Deutsche Version. Verfügbar unter www.piqinfo.ch [29.08.2019].

Venetz, M., Zurbriggen, C. L. A. \& Schwab, S. (2019). What do teachers think about their students' inclusion? Consistency of students' self-reports and teacher ratings. Frontiers in Psychology, 10, 1637. https://doi.org/10.3389/fpsyg.2019.01637

Wiesner, C., Schreiner, C., Breit, S. \& George, A. C. (2018). Evidenzorientierte Schulund Unterrichtsentwicklung: Preflectioning als Voraussetzung fur Entwicklung (Evidence-oriented development of schools and teaching: Preflectioning as a requirement for development). In C. Juen-Kretschmer, K. Mayr-Keiler, G. Orley \& I. Plattner (Hrsg.), transfer Forschung $\leftrightarrow$ Schule Heft 4-Schule 21st-Perspektiven der Schulentwicklung im 21. Jahrhundert (S. 95-111). Bad Heilbrunn: Klinkhardt.

Zurbriggen, C., Venetz, M., Schwab, S. \& Hessels, M. G. P. (2019). A psychometric analysis of the student version of the perceptions of inclusion questionnaire (PIQ). European Journal of Psychological Assessment, 35, 641-649. https://doi. org/10.1027/1015-5759/a000443 
Susanne Schwab, Dr., Prof. am Institut Zentrum für LehrerInnenbildung, Universität Wien sowie Extraordinary Prof. in der Research Focus Area Optentia, North-West-University, Vanderbijlpark, South Africa. Arbeitsschwerpunkte: Schulpädagogik mit besonderer Berücksichtigung sozialer, sprachlicher und kultureller Vielfalt, schulische Inklusion

susanne.schwab@univie.ac.at

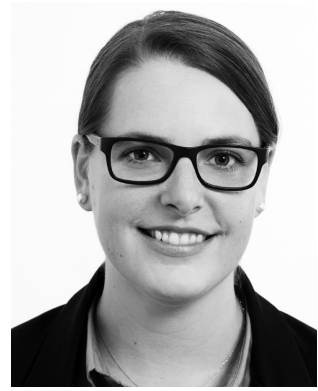

Matthias Huber, Dr., Univ.ass. und Lektor am Institut für Bildungswissenschaft und am Zentrum für LehrerInnenbildung der Universität Wien. Arbeitsschwerpunkte:

Bildung und Emotion, Lehr-Lern-Forschung, Bildungsverlaufsforschung, Pädagogische Epistemologie, Schulentwicklung, Mixed-Methods-Research

matthias.huber@univie.ac.at

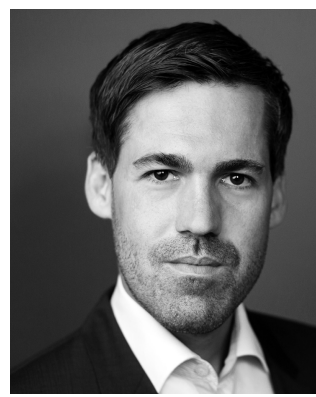

